\title{
Examining the influence of DPP4 inhibitors and sulphonyureas in combination with metformin on the Type II Diabetes gut microbiome: a pilot study
}

\author{
P. Cronin ${ }^{1,2}$, P.W. O'Toole ${ }^{2}$ and E.M. O' Connor ${ }^{1,2,3}$ \\ ${ }^{1}$ Department of Biological Sciences, University of Limerick, Limerick, Ireland, \\ ${ }^{2}$ APC Microbiome Ireland, University College Cork, National University of Ireland, Cork, Ireland and \\ ${ }^{3}$ Health Research Institute, University of Limerick, Limerick, Ireland
}

Type II Diabetes Mellitus (T2DM) has increased in prevalence globally over the last number of decades particularly in industrialised nations. This is primarily due to the Western diet, which is high in saturated fat and low in dietary fibre. Evidence has shown the gut microbiome to be altered in patients with T2DM when compared to healthy controls ${ }^{(1)}$. Given T2DM is characterized by elevated blood glucose levels due to insulin resistance and inadequate insulin secretion, a wide range of metabolic pathways are implicated by this pathogenesis ${ }^{(2)}$. As a result, numerous medications have been developed in order to combat this disease. Indeed, the mechanism of action of the most widely prescribed, first line antidiabetic therapy, metformin, has been shown to alter gut microbiota composition $^{(3)}$. Other commonly prescribed drugs include sulphonylurea and dipeptidyl peptidase 4 (DPP4) inhibitors (often prescribed in addition to metformin as second line therapies) which have been poorly studied in terms of potential microbiome impact.

Thus, we conducted a pilot study $(n=20)$ on T2D patients, all of whom were prescribed metformin in addition to either a DPP4 inhibitor $(n=6)$, a sulphonylurea $(n=8)$ or a combination of the latter two therapies $(n=6)$. Faecal samples were collected and the gut microbiota profiled using $16 \mathrm{~S}$ rRNA gene amplicon sequencing.

Beta-diversity measures (Weighted Unifrac - measure of inter-individual species diversity) revealed significant differences between all groups examined. Individuals prescribed sulphonylurea had lower alpha-diversity (Shannon Index - measure of species diversity between specific communities) while this group also differed when comparing the log2-fold differences of the most abundant amplicon sequence variants (representing sequence-based bacterial divisions).

This pilot study provides evidence that these medications may exert an influence on the gut microbiota and the efficacy of these medications when used with first line therapy may depend on microbiome interactions.

\section{References}

1. Qin J, Li Y, Cai Z, et al. (2012) Nature 490(7418), 55-60.

2. Saltiel AR \& Kahn CR (2001) Nature 414(6865), 799-806.

3. Wu H, Esteve E, Tremaroli V, et al. (2017) Nat Med 23(7), 50. 